\title{
Estimation of Walk-Run Transition Speed and Oxygen Consumption on Planets of Solar System
}

\author{
Hossien $H^{*}$, Kani MR, Gulstan SE and Nzar RA
}

Department of Physics, School of Science, University of Sulaimani, Kurdistan, Iraq

\begin{abstract}
The present work has analyzed and discussed the effect of gravitational force on the walk-run transition speed on the planets of the solar system. A walk-run transition speed at different gravity level has been calculated. Our results suggested that by increasing gravity level, the walk-run transition speed occurred at faster speed whereas the corresponding Froude numbers remain constant in normal and high gravities. The most significant effect of gravity on the Froude number was observed for the planets with gravity lower than the earth. In addition, the rates of oxygen consumption at the walk-run transition speed for these celestial objects have been predicted. The results showed that the rate of oxygen consumption for the planets are at the highest for those which have a gait transition at Froude number of greater than 0.5 .
\end{abstract}

Keywords: Froude numbers; Solar system; Walk-run transition; Oxygen consumption

\section{Introduction}

Humans have two main forms of locomotion which are walking and running whereas most of the quadrupeds have three forms of locomotion which are walk, trot and gallop. According to the classical definition, walking is a move at a regular step by lifting and setting down each foot in turn, which means that both feet will never be off the ground at once while in the running the move is faster, so that both feet leave the ground during each stride [1]. When somebody is walking, his/her walking speed is gradually increased and at a critical speed the action transfer to running state. Such a transition of movement happens because the running is more comfortable than walking in that speed, even though the person can still walk at that critical point. Although it is not entirely clear why human prefer to transit from one gait to another at a given speed and how this transition is affected by mechanics of walking and running. It has been reported that for human at a speed of faster than $2 \mathrm{~m} / \mathrm{s}$, metabolically walking is displeasing than running. This speed is almost the same as the observed gait transition speed, suggesting that there is a metabolic trigger for the walk-run transition i.e. there is an energy saving mechanism that causes the walkrun transition [2]. Despite of a number of studies on this topic [3-5], the influence of metabolic energy on the gait transition remains unclear. Studies on quadrupedal animals showed that the trot-gallop transition for horses have occurred at the minimum metabolic energy [6]. While other studies on the trot-gallop transition for horses [7] and walk-run transition for humans $[3,8,9]$ have reported that the gait transition occurred at slower speed than the predicted speed, based on metabolic energy concept. However, the metabolic cost per unit distance is found to be higher for running than walking at the transition speed, but the fluctuation of the metabolic energy per step is appeared to be the same for both gaits [8], indicating that the gait transition is not triggered by the metabolic energy.

Recent studies suggest that the gait transition should be interpreted mechanically instead of metabolically. A research has showed that the trot- gallop transition in horses occurs at different speeds but at the same level of the vertical ground reaction force (which is the reacting force to the one that the body centre of mass exerted via the body segments in contact with the ground) [7]. However it is reported that since the peak ground reaction forces increases (the transition from walking to running occurs), the gait transition in humans may not be interpreted by the same mechanism [10-12].

In addition, the effect of kinematic factor (maximum loading rate, braking and propulsive impulse, and braking and propulsive force peaks) on the gait transition during human locomotion is shown. It has been observed that only kinematics variable that may trigger the walkrun transition is the maximum ankle angular velocity. This variable increases with increasing speed of walking and decreases when running starts. It has been demonstrated that the walk-run transition towards the peak of a slope occurs at different speed, but at the same maximum ankle angular velocity. It has been speculate that the stress on the anterior tibialis muscle is reduced after the walk-run transition [13].

Although, the same case did not occur during run-walk transition. Walking speed as a function of leg length can be plotted by Parabola curves for adults. for $\mathrm{L}=0.92 \mathrm{~m}$ maximal pendulum-like energy exchange as in the spontaneous and optimal walking speed occurs in Froude number equal to 0.25 and walk-run transition speed occurs in Froude number equal to 0.5 [14].

\section{Model and Theory}

We have used inverted pendulum mechanical model to describe Bipedal walking. The model assumed that the total body mass is concentrated in one point and legs are a rigid strut [14]. According to this model, no mechanical energy for continuing motion is required and fluctuation of kinetic energy and potential energy are equals and precisely 180 degree out of phase [10].

The main force that determines the movement of invertedpendulum-like during walking is the gravity that should be equal to the centripetal force to maintain the body motion about the stance of the

*Corresponding author: Hossien $\mathrm{H}$, Department of Physics, School of Science, University of Sulaimani, Kurdistan, Iraq, Tel: 964-053-330-10; E-mail: Hossien.Hossieni@univsul.ne

Received July 16, 2014; Accepted July 29, 2014; Published July 31, 2014

Citation: Hossien H, Kani MR, Gulstan SE, Nzar RA (2014) Estimation of WalkRun Transition Speed and Oxygen Consumption on Planets of Solar System. Astrobiol Outreach 2: 119. doi: 10.4172/2332-2519.1000119

Copyright: (c) 2014 Hossien H, et al. This is an open-access article distributed under the terms of the Creative Commons Attribution License, which permits unrestricted use, distribution, and reproduction in any medium, provided the original author and source are credited. 


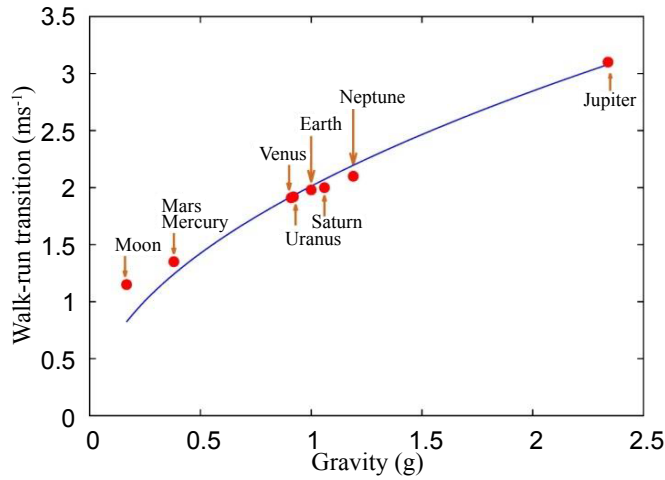

Figure 1: Walk-run transition speed at different gravitation force for the planets of the solar system.

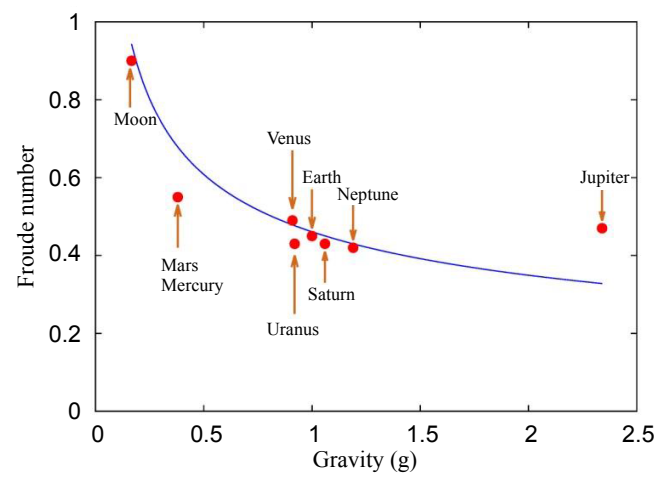

Figure 2: Calculated values of Froude number at the walk-run transition speed for different gravitation force in the planets of the solar system

foot. i.e. when $\mathrm{F}$ is not provided, there is a risk of takeoff and the model fails. The centripetal force (F) can be described as follow

$$
F=\frac{m v^{2}}{L}
$$

Where $\mathrm{m}$ is mass of the body, $\mathrm{L}$ is leg length and $\mathrm{v}$ is forward speed.

The centripetal force suggests that maximum walking speed up to a limit that a circular path maintained is mainly controlled by the gravity.

The ratio between centripetal force and gravity can be defined as a Froude number (Fr) [15]:

$$
F r=\frac{\left(m v^{2}\right) / L}{m g}=\frac{v^{2}}{g L}
$$

Equation 2 indicates that the above Froude number of 1, walking is impossible. This is because that would mean that the gravity is weaker than the required centripetal force need to maintain the body on the circular arc [16].

Interestingly, it can be seen that the relation between $\mathrm{Fr}$ and $\mathrm{v}$ is independent on the body size but it is mainly depends on the leg length and the gravity. The observation on bipeds (including human and birds) suggested that the walk-run transition occur at Fr of 0.5 [15-18]. The relationship between Froude number and walk-run transition has been investigated by a number of studies $[16,19]$.

Simulate reduced gravity instrument is used to study gravity effect on walk-run transition speed on nine persons ( 7 men and 2 women) who had the same leg length $\mathrm{L}=0.89 \mathrm{~m}$, and the same mass. The results indicated that at low values of gravity, the walk-run transition occurred at lower speed. The increase of the transition speed was found to be more significant above the gravity of $3.92 \mathrm{~m} / \mathrm{s}^{2}$. It has also been observed that below this gravity level the prediction of inverted pendulum model for equal Froude number did not hold. This was attributed to the influence of acceleration due to moving arms and legs in the walking process [16].

In an attempt to study the prediction of inverted pendulum model for the gait transition on the planets of the solar system, the optimal walking and walk-run transition speed at Froude numbers of 0.25 and 0.5 has been shown respectively while the leg length was kept constant. The results showed that the optimal walking speed and walk-run transition speed of a celestial objects with $16 \%$ of the earth's gravity (Moon) were about 0.6 and $0.8 \mathrm{~m} / \mathrm{s}$, respectively. This is about $40 \%$ of those on the earth [19].

With the above studies on the influence of gravity on the walkrun transition speed, it is important to clarify how human can move on different planets. In addition, it is interesting to find out whether the walk-run transition will occur at the same Froude number that has been predicted for the earth.

Furthermore, only a limited number of studies have reported the variation of oxygen cost at the walk-run transition speed. Our study has calculated the breath -by- breath rates of oxygen consumption relating to the Froude numbers at different leg length. It has been shown that the amount of oxygen consumption decreased by increasing the Froude number. At the Froude number of 0.25, the amount of $\mathrm{O}_{2}$ cost is minimum. Above this value, the $\mathrm{O}_{2}$ cost starts to increase. It has been noticed that where the oxygen consumption was minimum, walking was optimal i.e. the exchange of potential energy to kinetic energy is maximum [20].

Another purpose of our study is to reanalyze whether the changes of the gravity level on other planets of the solar system affect the prediction of inverted pendulum model for the Froude number at the walk transition ( $\mathrm{Fr}=0.5$ ) (Ref). Moreover, the variation of oxygen consumption at the predicted walk-run transition on the planets of the solar system is taken into our calculations.

\section{Results and Discussion}

In this section, an estimation of Walk-run transition speed and Froude number, and variations of Oxygen consumption at the walk-run transition speed for the planets of the solar system are shown as follows:

\section{Walk-run transition speed and froude number}

The planets of the solar system have different gravity which is between 0.39 to 2.34 times the gravity of the earth. By using previous literature data and interpolation and extrapolation algorithms, the walk-run transition speed of the planets of the solar system has been obtained as shown in Figure 1.

The calculated values of the walk-run transition speed and the gravity level were substituted in Eq. 1 and the relevant Froude numbers have been calculated for each planet of the solar system as demonstrated in Figure 2.

Figure 1 shows that by reducing the gravity level, the walk-run transition occurs at slower speed. The walk-run transition speed of the planet with 2.3 times higher gravity than the earth (such as Jupiter) is about 1.5 times higher than those of the earth. 


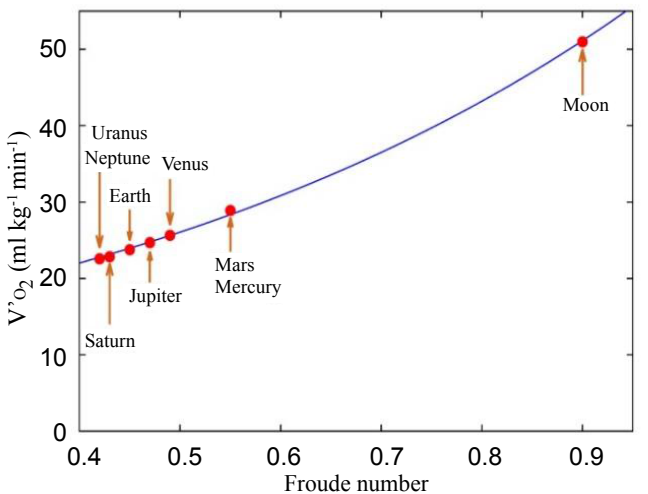

Figure 3: Rate of oxygen consumption at various Froude number on the planets of the solar systems.

Figure 2 display that for the planets with gravity levels higher than the earth, the walk-run transition occurs at Froude number around 0.5, while for the planets with lower gravity than the earth, this transition occur at higher Froude number. This result indicates that for the planets of lower gravity than the earth, the prediction of the gait transition at Froude number of 0.5 is not hold.

In Figure 2, it can be seen that only the value of the Moon is higher than 0.5 , while Mars, Mercury, Venus and Uranus, are compatible with Froude $=0.5$.

The gait transition at Froude number is greater than 0.5 for the low gravity level have also been reported by others and attributed to neglecting the acceleration produced by swinging limbs. It has been shown that the acceleration produced from swinging of arms and legs are relatively high at the low gravity level and by increasing the gravity, the influence of limbs acceleration become insignificant [16].

\section{Oxygen consumption at the walk-run transition speed}

The rate of oxygen consumption at the walk-run transition speed for the other planets of the solar system have been calculated by the following regression formula [17]:

$$
v^{\prime} O_{2}=29.8\left(F r^{2}\right)+19.8(\mathrm{Fr})+0.91
$$

The calculated values of the rate of oxygen consumption are plotted in Figure 3. It can be observed that the oxygen consumption for the planets which have the gait transition at Froude number greater than 0.5 is at the highest. This is consistent with the previous study [20] and suggesting that the walk-run transition on these planets requires higher metabolic cost.

\section{Conclusions}

We used the previous literature data and inverted pendulum model to calculate the effect of changing gravity on walk-run transition speed and Froude number for bipedal animals on the planets of the solar system. The results have shown that the walk-run transition for the planets with higher gravity than the earth, the transition achieves at the higher speed but at similar Froude numbers. The prediction of occurring walk-run transition at Froude number of 0.5 for the planets with low gravity level did not hold.

In addition, other previous literature data has been used here to calculate the rate of oxygen consumption at Froude number, at which the gait transitions were observed. It has been noticed that the rate of oxygen consumption were at the highest value for the low gravity celestial object such as the moon while this value did not have a significant change between the high and average gravity planets of the solar system.

\section{References}

1. Hildebrand $M$ (1985) Walking and running. In Functional Vertebrate Morphology. Belknap Press, USA

2. Margaria R (1976) Biomechanics and Energetics of Muscular Exercise. Clarendon Press, USA.

3. Hreljac A (1993) Preferred and energetically optimal gait transition speeds in human locomotion. Med Sci Sports Exerc 25: 1158-1162.

4. Mercier J, Le Gallais D, Durand M, Goudal C, Micallef JP, et al. (1994) Energy expenditure and cardiorespiratory responses at the transition between walking and running. Eur J appl Physiol 69: 525-529.

5. Minetti AE, Ardigo LP, Saibene F (1991) The transition between walking and running in humans: metabolic and mechanical aspects at different gradients. Acta physiol scand. 150: 315-323.

6. Hoyt DF, Taylor CR (1981) Gait and energetics of locomotion in horses. Nature 292: 239-240.

7. Farley CT, Taylor CR (1991) A mechanical trigger for the trot-gallop transition in horses. Science 253: 306-308.

8. Minetti, AE, Ardigo LP, Saibene F (1994) The transition between walking and running in humans: metabolic and mechanical aspects at different gradients. Acta physiologica scandinavica 150: 315-323.

9. Brisswalter J, Mottet D (1996) Energy cost and stride duration variability a preferred transition gait speed between walking and running. Canadian Journal of Applied Physiology 21: 471-480.

10. Cavagna GA, Heglund NC, Taylor CR (1977) Mechanical work in terrestria locomotion: two basic mechanisms for minimizing energy expenditure. Am J Physiol 233: R243-R261.

11. Hreljac A (1993) Determinants of the gait transition speed during human locomotion: kinetic factors. Gait Post 1: 217-223.

12. Nilsson J, Thorstensson A (1989) Ground reaction forces at different speeds of human walking and running. Acta Physiologica Scandinavica 136: 217-227.

13. Hreljac A (1995) Determinants of the gait transition speed during human locomotion: kinematic factors. J Biomech. 28: 669-677.

14. Franco S, Alberto EM (2003) Biomechanical and physiological aspects of legged locomotion in humans. Eur J Appl Physiol 88: 297-316.

15. Alexander RM (1977) Mechanics and scaling of terrestrial locomotion. In Scale Effects in Animal Locomotion Academic Press, New York.

16. Rodger K, Domingo A, Daniel PF (1997) Effect of reduced gravity on the preferred walk-run transition speed. The Journal of Experimental Biology 200: 821-826.

17. Alexander RM (1989) Optimization and gaits in the locomotion of vertebrates Physiol Rev 69: 1199-1227.

18. Hreljac A (1995) Effects of physical characteristics on the gait transition speed during human locomotion. Human Mov Sci 14: 205-216

19. Alberto EM (2001) Walking on other planets. Nature 409: 467-469.

20. Françoise L, Yuri PI, Bengoetxea A, Ana-Maria C, Bernard D, et al. (2011) Optimal walking speed following changes in limb geometry. The Journal of Experimental Biology 214: 2276-2282. 